\title{
iWalk: Uma Solução para Medição e Análise da Caminhabilidade de Cidades com Portais de Dados Abertos
}

\author{
Vinícius Gonçalves Pisco ${ }^{1}$, Humberto Torres Marques-Neto ${ }^{1}$ \\ ${ }^{1}$ Departamento de Ciência da Computação \\ Instituto de Ciências Exatas e Informática \\ Pontifícia Universidade Católica de Minas Gerais (PUC Minas) \\ Rua Walter Ianni, 255 - São Gabriel - 31980-110 - Belo Horizonte/MG \\ vgpiscoesga.pucminas.br, humbertodpucminas.br
}

\begin{abstract}
Understanding the interest of pedestrians movements in a region is an important task to improve the effectiveness of mobile applications and is directly related to the quality of life in urban spaces. In this sense, this work presents $i$ Walk, a solution based on the calculations of the walkability index present in the literature. Through urban computing and using open data, with iWalk, it is possible to measure and analyze the quality of urban infrastructure in a scalable way, without the need for on-site visits. The results of the simulations indicate that the proposed solution is able to present an efficient model for the analysis of walkability indicators, using open data available in government portals.
\end{abstract}

Resumo. Entender o interesse da movimentação de pedestres por uma região é uma tarefa importante para melhorar a efetividade de aplicações móveis e possui relação direta com a qualidade de vida dos espaços urbanos. Neste sentido, este trabalho apresenta o iWalk, uma solução baseada nos cálculos de índice de caminhabilidade presentes na literatura. Por meio da computação urbana e utilizando dados abertos, com o iWalk, é possível medir e analisar a qualidade das infraestruturas urbanas de maneira escalável, sem a necessidade de visitas in loco. Os resultados das simulações indicam que a solução proposta consegue apresentar um modelo eficiente para a análise de indicadores de caminhabilidade, utilizando dados abertos disponíveis em portais governamentais.

\section{Introdução}

Com o rápido crescimento da população urbana mundial, cidades do mundo todo começaram a procurar soluções que permitissem interligar os sistemas de transporte, promover o uso misto do território e prover serviços urbanos de alta qualidade, com efeitos positivos na qualidade de vida da população. Muitas das novas abordagens relacionadas aos serviços urbanos se basearam na tecnologia e ajudaram a criar o conceito de Cidades Inteligentes [Albino et al. 2015].

A aplicação das Tecnologias da Informação e da Comunicação (TIC) em Cidades Inteligentes promove o desenvolvimento de uma área de pesquisa denominada Computação Urbana, a qual pode ser compreendida como o suporte computacional para a construção de ambientes urbanos que oferecem serviços avançados aos cidadãos. A Computação Urbana é o processo de aquisição, integração e análise de uma quantidade 
grande e heterogênea de dados gerados por diferentes fontes como sensores, dispositivos, veículos, prédios e pessoas, para tratar de questões críticas para as cidades, como poluição, consumo de energia e mobilidade humana [Zheng et al. 2014].

Neste contexto de Computação Urbana, a Mobilidade Inteligente surgiu como uma solução para os problemas causados pelo uso intenso dos modos motorizados de transporte e pela negligência aos meios transporte ativos, como pedalar e caminhar. Os benefícios de adotar modos de transporte mais sustentáveis são geralmente apreciados pelas pessoas, mas as várias abordagens para mudar o paradigma da mobilidade falharam devido aos fatores complexos que as impedem de adotarem mudanças comportamentais. Entender como promover os meios de transporte ativos é um componente essencial para que as políticas públicas alcancem um processo de desenvolvimento mais sustentável [Fonseca et al. 2019]. A capacidade de caminhar (walkability, em inglês), geralmente é definida como uma questão multidimensional em que estas dimensões são mensuráveis individualmente ou combinados em um índice ou indicador, formando assim o índice de caminhabilidade [Forsyth 2015].

$\mathrm{Na}$ perspectiva de responder à lacuna existente em mecanismos computacionais que buscam o entendimento dos fatores que influenciam diretamente na caminhabilidade do espaço público, entende-se que é necessário aprofundar o estudo dos índices existentes para se mensurar a caminhabilidade de um determinado espaço urbano. Isto posto, este estudo tem como objetivo apresentar o iWalk, uma solução capaz de analisar os indicadores presentes em bases de dados abertos que compõem um índice de caminhabilidade. Utilizando uma escala de 0 a 100, o iWalk determina a caminhabilidade de um determinado espaço público, considerando o indicador calculado, e auxilia a prever a possibilidade de uso de um determinado espaço urbano por pedestres, ajudando no direcionamento da tomada de decisão em projetos de aplicações móveis e redes sem fio.

Para a elaboração desta solução, foi feito um estudo de caso para a cidade de Lisboa, Portugal, realizando a análise e o cálculo do indicador topográfico de declividade, que é um dos indicadores que compõem um índice de caminhabilidade. Nossa proposta foi validada por meio da comparação dos resultados obtidos no cálculo de um dos indicadores de caminhabilidade pelo iWalk com os resultados obtidos em um trabalho conhecido da literatura, que desenvolveu um índice de caminhabilidade, formado por 27 indicadores, para a cidade de Belo Horizonte.

Este estudo está organizado da seguinte maneira: após esta introdução, a Seção 2 apresenta o referencial teórico que fundamenta este estudo. Em seguida, na Seção 3, apresentamos como são feitos os cálculos de caminhabilidade. Logo após, na Seção 4, é apresentada nossa proposta, chamada de iWalk. Na Seção 5, é feita uma validação da proposta e na Seção 6, as conclusões e trabalhos futuros deste estudo.

\section{Trabalhos Relacionados}

Considerando as evidências de que ambientes urbanos desencorajam caminhadas e outras atividades físicas, foram criados vários índices de caminhabilidade para descrever a área caminhável, combinando aspectos do ambiente construído que promovem ou inibem o caminhar no espaço urbano [Glazier et al. 2012]. O desenvolvimento da caminhabilidade e das infraestruturas que a favorecem, geralmente recebem menos investimentos do que o adequado. Portanto, melhorias na capacidade de caminhar podem também fornecer um 
alto retorno econômico do investimento [Quercia et al. 2015]. Além disso, o desenvolvimento de sistemas que permitam visualização e análises dos dados disponibilizados por meios de portais de dados abertos é de grande valia para o entendimento de padrões e formulação de novos questionamentos [Xavier 2019].

[Wang and Song 2015] aproveitaram as vantagens de um enorme conjunto de dados de telefonia móvel sobre mobilidade humana e informações de redes sociais, para explorar o impacto dos padrões de mobilidade humana na rede social subjacente. Neste trabalho de 2015, concluíram que a mobilidade humana desempenha um papel importante na formação das propriedades estruturais locais e globais de redes sociais.

Os autores de [Quercia et al. 2015] exploraram a possibilidade de utilizar dados de redes sociais como Flickr ${ }^{1}$ e Foursquare ${ }^{2}$ para identificar automaticamente ruas seguras e fáceis de percorrer. Descobriram que ruas inseguras tendem a ser fotografadas durante o dia, enquanto as ruas mais tranquilas geralmente são marcadas com palavras-chave relacionadas à mobilidade.

Em [Frank et al. 2005], acelerômetros foram distribuídos para 357 adultos de modo a acompanhar, por 2 dias, a atividade física de cada participante. Foram controlados aspectos ambientais ao redor da casa de cada um. As medidas de combinação de uso do solo, densidade residencial e densidade de interseção tiveram uma relação positiva com o número de minutos de atividade física moderada por dia. Do total de pessoas que receberam o acelerômetro, $37 \%$ das pessoas residentes em locais com o índice de caminhabilidade mais alto atingiram os 30 minutos de atividade física recomendado, um número 2,4 vezes maior do que aqueles que residiam em locais com um índice mais baixo.

[Mantri 2008] e [Bhadra et al. 2015] realizaram abordagens baseadas em Sistemas de Informação Geográficas (GIS) para realizar avaliações objetivas e mensurar a caminhabilidade do espaço urbano e em [Papageorgiou et al. 2020] os autores investigaram até que ponto as pessoas estão preparadas para usarem um aplicativo de navegação capaz de aprimorar sua experiência de caminhada, ajudando a se tornarem mais saudáveis e ao mesmo tempo melhora as condições ambientais. Os resultados mostraram que, além das informações de navegação, fornecer recompensas motiva as pessoas a andarem mais.

Já em [Cardoso et al. 2019], os autores desenvolveram um índice de caminhabilidade capaz de avaliar um determinado trecho urbano da cidade de Belo Horizonte. Analisando os resultados, os autores concluíram que os trechos que obtiveram as piores notas possuem um uso do solo predominantemente residencial e são caracterizados por vias de grande circulação de veículos, em condições desfavoráveis à circulação de pedestres, dimensões estreitas de calçada, falta de sinalização, entre outros.

Neste trabalho, a solução proposta iWalk engloba as principais técnicas descritas na literatura para realizar o cálculo da caminhabilidade do ambiente. Esta proposta se difere por utilizar uma base de dados abertos conectados para a aplicação do índice de caminhabilidade. O cálculo do índice do seguimento realizado em [Cardoso et al. 2019] é utilizado como base de comparação para aferir a qualidade da proposta e validar os resultados obtidos pelo iWalk.

\footnotetext{
${ }^{1}$ http://www.flickr.com

${ }^{2}$ http://www.foursquare.com/
} 


\section{Mensurando a Caminhabilidade}

Conforme serão apresentados nesta seção, vários índices foram desenvolvidos para medir a capacidade de caminhar, combinando aspectos do ambiente que promove ou inibe a caminhada. Esses índices geralmente incluem medidas, chamadas de indicadores de caminhabilidade, como declividade da rua, densidade de habitações residenciais, densidade de interseção (uma medida de conectividade das ruas), densidade ou proximidade de destinos para caminhadas e combinação de uso da terra e mostraram associações positivas com a frequência ou duração da atividade física e associações negativas com o peso corporal em vários ambientes. É comum que os pesquisadores adaptem o desenvolvimento de um índice baseado na disponibilidade e qualidade dos dados do ambiente construído para suas áreas de estudo. Alguns índices dependem de dados de diversas variáveis, como avaliação de imposto de propriedade em nível de lote e a combinação do uso da terra [Glazier et al. 2012].

Os índices de caminhabilidade descrevem com sucesso o ambiente de caminhada em muitas cidades e ajudam no entendimento dos fatores que promovem ou inibem a caminhabilidade, mas devido a pequenas amostras ou falta de dados, muitos estudos realizados anteriormente não foram capazes de analisar adequadamente como as pessoas com diferentes necessidades de mobilidade podem ser afetadas pela capacidade de caminhar de sua vizinhança. A capacidade de caminhar por um determinado espaço público pode ser entendida como uma combinação entre os desejos dos residentes e expectativas para os tipos de destinos, a vontade de percorrer uma determinada distância e a qualidade do percurso pretendido [Manaugh and El-Geneidy 2011].

O índice de caminhabilidade desenvolvido por [Frank et al. 2005] tem sido usado para diferentes escalas geográficas ao redor de residências ou centros comerciais específicos, pois apoia a hipótese de que o desenho das infraestruturas urbanas está significativamente associado com níveis moderados de caminhabilidade. Este trabalho de 2005 sustenta a justificativa para o desenvolvimento de políticas que promovam níveis mais elevados de combinação de uso da terra, densidade residencial, segurança, atratividade e conectividade de ruas como intervenções que promovem a caminhabilidade pelo espaço público e que podem ter benefícios duradouros para a saúde e qualidade de vida da população.

Em uma abordagem muito semelhante, [Kuzmyak et al. 2006] ponderaram dados de varejo em distância, importância e conveniência dentro um conjunto de destinos possíveis. Os destinos utilizados diariamente, como farmácias e supermercados, receberam um pontuação de importância maior do que os utilizados esporadicamente, como centros de esportes ou casas noturnas.

Os Sistemas de Informação Geográfica (GIS), como o iWalk, podem ser utilizados para medir objetivamente as características do ambiente construído que podem influenciar na caminhabilidade. [Leslie et al. 2007] descreveram um índice de caminhabilidade desenvolvido utilizando os dados espaciais disponíveis. O índice era usado para gerar uma base de amostragem estratificada para a seleção de famílias de 32 comunidades. Os dados GIS têm potencial para serem usados para construir medidas de atributos ambientais e desenvolver índices de caminhabilidade para cidades, regiões ou comunidades locais. A Figura 1, apresenta um modelo simplificado de um sistema GIS semelhante ao utilizado pelo iWalk, cortado verticalmente através de camadas de dados para análise em áreas es- 
paciais de localização conhecida. Cada camada do sistema GIS representa um indicador de caminhabilidade presente em uma base de dados específica.

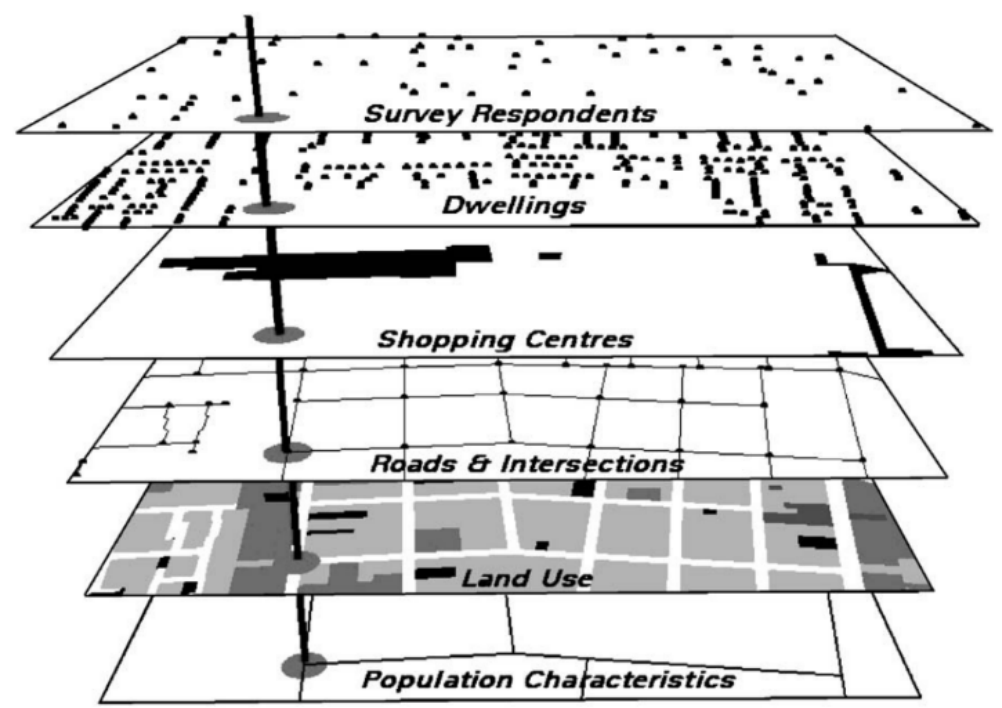

Figura 1. Modelo simplificado de um sistema GIS [Leslie et al. 2007].

A expansão das políticas de dados abertos conectados (LOD) nas esferas municipal, estadual e nacional é uma porta de entrada eficaz para a estratégia tecnológica e favorece o desenvolvimento de sistemas GIS. Com os portais governamentais de dados abertos, os governos podem colaborar para que pesquisadores melhorem a eficiência das operações nas cidades e ajudem a aconselhar sobre políticas baseadas em dados [Schenk 2015].

Neste sentido, apresentamos na seção seguinte uma solução para realizar o cálculo do índice de caminhabilidade utilizando um sistema de informações geográficas presentes em portais governamentais de dados abertos.

\section{4. iWalk}

Esta seção apresenta a solução chamada iWalk, a qual foi pensada para oferecer uma abordagem capaz de calcular indicadores de caminhabilidade utilizando fontes de dados abertos, na qual os interessados em dados de caminhabilidade podem calcular e/ou consultar indicadores que suportem suas decisões seja de cunho governamental ou pessoal. Com ela, é possível extrair conhecimento sobre os diversos indicadores que compõem um índice de caminhabilidade em diferentes escalas possíveis, sejam elas por logradouro, bairro, regional ou a cidade inteira. Para esta proposta, é importante ressaltar a dificuldade de se realizar um cálculo de índice de caminhabilidade apenas com visitas in loco ou utilizando de extração de conhecimento das bases de dados públicas de maneira manual. Este tipo de cálculo em que se torna necessária a visita presencial à área de estudo, por muitas vezes não é escalável para uma grande área de cobertura, devido à imensa demanda por processos manuais e à grande possibilidade de ocorrerem erros de cálculo devido a esse processo humano. Com o iWalk, por meio do uso da computação urbana, é possível determinar os parâmetros e os dados que irão compor o índice, desde que os dados estejam disponíveis na grande rede. 


\subsection{Arquitetura}

O iWalk é um conjunto de funcionalidades desenvolvidas em Python 3 para leitura de dados abertos no formato GeoJSON, que é um formato de padrão aberto, baseado no formato JSON, projetado para representar características geográficas simples, juntamente com seus atributos não geográficos. A Figura 2 apresenta o fluxo de execução do iWalk para o cálculo de cada indicador de caminhabilidade e as seções seguintes apresentam cada parte do fluxo e suas transições. Para a execução do fluxo de dados do iWalk foi realizado um estudo de caso para a cidade de Lisboa, pois a capital portuguesa possui um portal de dados abertos com os dados pertinentes ao cálculo de indicadores de caminhabilidade.

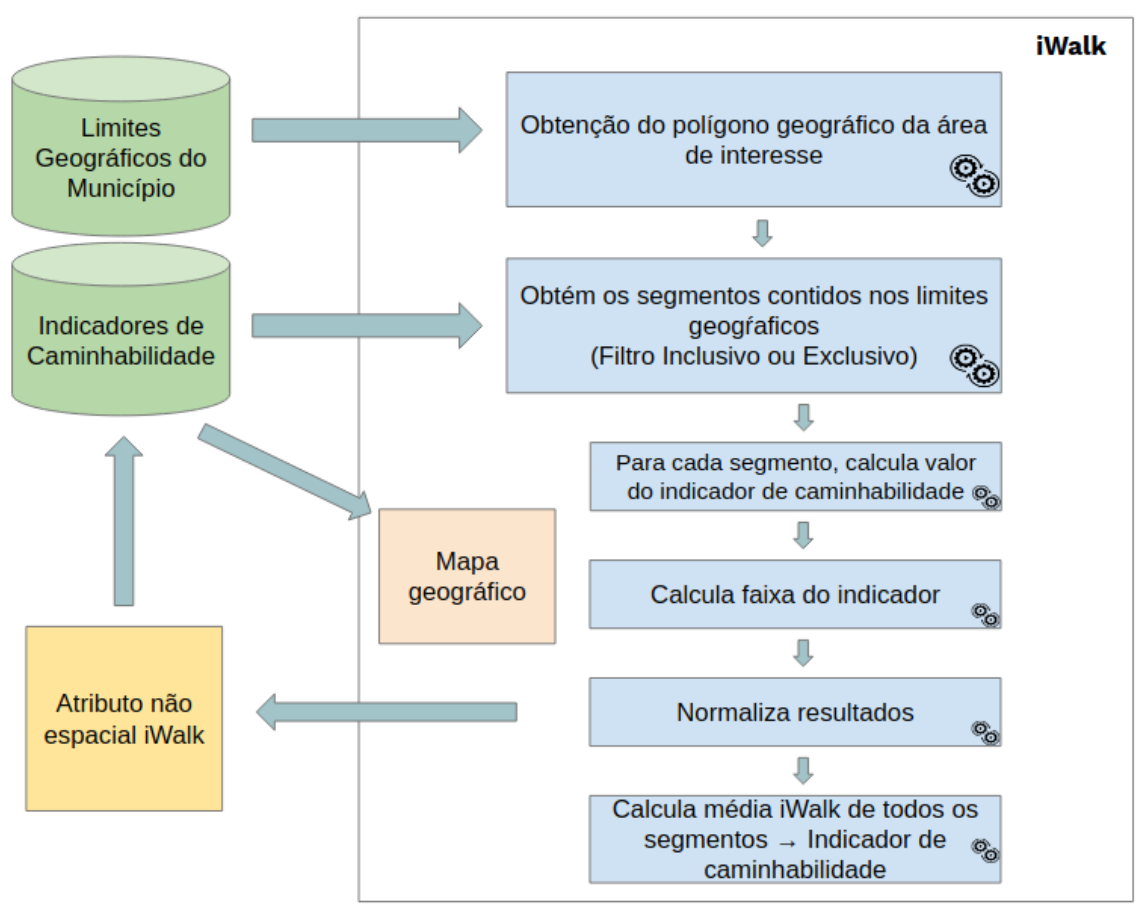

Figura 2. Fluxo de execução do iWalk.

\subsection{Parâmetros de entrada e saída}

O conjunto de parâmetros de entrada é formado por um ou mais conjuntos de dados extraídos dos portais de dados abertos, a escrita de uma função para o cálculo do indicador de caminhabilidade desejado, que leva em consideração os atributos da base GeoJSON, e uma função para determinar a faixa em que o cálculo realizado pela função anterior será inserido. Como saída, o iWalk fornece um mapa geográfico com a representação em cores dos indicadores calculados e o indicador de caminhabilidade calculado para a região objeto do estudo, em uma escala de 0 a 100. A escala de cores apresentada no mapa geográfico vai do verde ao vermelho, onde o verde representa a melhor caminhabilidade e vermelho, a pior. 


\subsubsection{Conjuntos de Dados}

Uma constante nos portais de dados abertos é a presença de um primeiro conjunto de dados que contém os dados dos limites geográficos das cidades. Por exemplo, no estudo de caso realizado neste trabalho para a cidade de Lisboa, é possível encontrar no portal de dados abertos da capital portuguesa ${ }^{3}$ o conjunto de dados denominado Freguesias-2012, que contém os dados referentes aos limites geográficos dos bairros de Lisboa. Utilizamos este conjunto de dados para extrair o polígono do bairro de Belém. Este primeiro conjunto tem como função principal estabelecer os limites geográficos da área de estudo para que seja realizada uma filtragem dos conjuntos de dados seguintes que possuem as informações de indicadores de caminhabilidade desejadas.

Inserimos então um segundo conjunto de dados, presente no mesmo portal de dados abertos de Lisboa, denominado Declive Longitudinal da Rede Viária. Este conjunto possui as informações de declividade das ruas da região a ser estudada.

\subsubsection{Atributos Espaciais e Não-Espaciais do Conjunto de dados}

O conjunto de dados representado pelo arquivo GeoJSON possui atributos espaciais e nãoespaciais. Os atributos espaciais são aqueles que possuem as características geográficas do segmento ou área contidas no registro. Estes atributos são representados por figuras geométricas como polígono, uma reta ou ponto e possuem as informações geogŕáficas de latitude e longitude dos pontos que compõem estas figuras geométricas. Os atributos não espaciais são aqueles que representam as propriedades do registro contido na base de dados, como por exemplo nome da rua, comprimento do segmento, altura e distância de subida e descida do segmento, entre outros.

\subsubsection{Polígono Geográfico da Área de Interesse}

Na primeira transição do iWalk, é realizada a extração do polígono geográfico da área de interesse. Este polígono é apresentado nos atributos espaciais da primeira base GeoJSON descritos nas seções anteriores. Estes atributos podem ser dos tipos Polygon ou MultiPolygon do pacote Shapely do Python 3. O iWalk realiza então uma filtragem na base de dados para selecionar o polígono desejado e carregá-lo em uma variável para as próximas transições do sistema.

\subsubsection{Segmentos Contidos nos Limites Geográficos}

Na segunda transição, o iWalk realiza mais uma filtragem de dados. Desta vez, utiliza a segunda base de dados descrita mais acima e o polígono geográfico da área de interesse descrito na seção anterior para determinar quais segmentos de interesse da base de dados estão contidos no polígono. No estudo de caso realizado, os segmentos são trechos de ruas que estão representados nos arquivos GeoJSON. O filtro utilizado pode ser do modo

\footnotetext{
${ }^{3}$ http://lisboaaberta.cm-lisboa.pt/
} 
exclusivo ou inclusivo e significam, respectivamente, que se considera o segmento pertencente ao polígono se todos os atributos espaciais do segmento estão contidos dentro do polígono ou se apenas parte dos atributos estão contidos. Os atributos espaciais a serem filtrados podem ser dos tipos Polygon, MultiPolygon, LineString, MultiLineString e Point.

\subsubsection{Cálculo do Indicador de Caminhabilidade por Segmento}

Na próxima transição do iWalk, é calculado o indicador de caminhabilidade de cada segmento extraído pela filtragem descrita na seção anterior. Nesta transição, podem ser considerados os atributos espaciais e não espaciais dos segmentos. Este cálculo pode variar de acordo com a base de dados inserida no iWalk e depende do tipo de indicador que se busca e dos tipos de atributos não espaciais contidos na base de dados, sendo considerado assim, um parâmetro de entrada do iWalk. O cálculo do indicador de caminhabilidade pode ser, por exemplo, o cálculo da área do segmento de acordo com o seu tipo, a presença ou não do atributo não espacial do segmento a ser analisado, a distância do atributo para um determinado ponto de referência, o cálculo do valor do atributo não espacial ou de uma combinação deles. No exemplo do estudo de caso da cidade de Lisboa, a base de dados Declive Longitudinal da Rede Viária possui atributos não espaciais que contém os dados da declividade do segmento. Neste caso, o iWalk realiza o cálculo do valor do indicador de caminhabilidade considerando os seguintes atributos não espaciais com unidade de medida em metros: LenDown, que possui o comprimento do segmento de rua que apresenta declividade negativa (descida); LenUp, o comprimento que apresenta a declividade positiva (subida); HDown, que possui a altura total de descida e $H U p$, a altura total de subida. Utilizando a função que foi escrita como parâmetro de entrada do iWalk, calculamos o valor indicador de caminhabilidade de acordo com a Equação 1 para obter Dec, o percentual de inclinação média do segmento de rua:

$$
D e c=100 \times \frac{(H U p / \text { LenUp })+(\text { HDown } / \text { LenDown })}{2}
$$

Para evitar a divisão por zero, caso LenUp ou LenUp possuam valor zero, o termo da equação que possui esse atributo, HUp/LenUp ou HDown/LenDown é considero zero e não é feita a divisão por 2 apresentada na equação.

\subsubsection{Cálculo das Faixas de Caminhabilidade}

Na transição seguinte, o valor do indicador de caminhabilidade calculado pela função descrita na seção anterior é inserido dentro de uma faixa de valores de acordo com a função descrita como parâmetro de entrada do iWalk. Por exemplo, segundo a norma brasileira para acessibilidade a edificações, mobiliário, espaços e equipamentos urbanos, descrita em [Abnt 2015], a inclinação longitudinal da superfície caminhável deve ser inferior a $5 \%$. Inclinações iguais ou superiores a 5\% são consideradas rampas e devem possuir inclinação máxima de 8,33\%. Acima de 8,33\%, pessoas com dificuldade de locomoção, como cadeirantes, tem dificuldade de circulação com autonomia.

Nesse sentido, escrevemos a função do cálculo da faixa de caminhabilidade, que recebe como parâmetro o indicador de caminhabilidade calculado pela função descrita 
na seção anterior $(D e c)$ e retorna a faixa em que este valor calculado se encontra. Neste exemplo, os valores retornados pela função que calcula as faixas de caminhabilidade podem variar de 1 até 3 da seguinte maneira:

1. Se a declividade média for menor que $5 \%$ com a sensação de rua plana.

2. Se a declividade média estiver entre $5 \%$ e $8,33 \%$ com a sensação de uma leve declividade.

3. Se a declividade for acima de 8,33\% com a sensação de uma forte declividade, que prejudica a caminhabilidade.

\subsubsection{Função de Normalização}

Como a quantidade de faixas de caminhabilidade retornadas pela função descrita na seção anterior pode variar de acordo com o indicador calculado, é necessário realizar uma normalização desses valores para que todos os indicadores calculados pelo iWalk sejam representados sempre em uma escala de 0 a 100, onde 0 representa a pior caminhabilidade e 100 a melhor. Esta normalização é necessária para que o cálculo de qualquer indicador de caminhabilidade, não só o indicador de declividade, sejam representados na mesma escala. Por exemplo, no estudo de caso do indicador topográfico de declividade, é possível obter 3 resultados distintos que podem variar de 1 a 3 , conforme descrito na seção anterior. Em outros casos, para calcular outros indicadores que não seja o indicador de declividade, o número de faixas podem variar de acordo com o indicador que se busca, podendo variar, por exemplo, de 1 a 4 ou de 1 a 5. Neste sentido, em [Eastman and Jiang 1996] é proposta uma equação para este tipo de normalização de dados multi-critério, em que o número de critérios ou faixas podem variar. A Equação 2 é então utilizada para obtermos o indicador de caminhabilidade normalizado (IC) de cada segmento. Nesta equação, o Rmax e Rmin são, respectivamente, os valores máximo e mínimo das faixas de caminhabilidade (por exemplo, 1 e 3) e o Ri é o valor da faixa obtido pela função descrita na seção anterior.

$$
I C=100 \times \frac{(\operatorname{Rmax}-R i)}{(\operatorname{Rmax}-R \min )}
$$

\subsubsection{Atributo Não Espacial "iWalk"}

Na transição seguinte, após a normalização do valor calculado para o indicador de caminhabilidade descrita na seção anterior, os valores normalizados das faixas de caminhabilidade, representados na escala de 0 a 100 para cada segmento calculado, são inseridos na base da dados utilizada como parâmetro de entrada no formato de um novo atributo não espacial do segmento denominado $i$ Walk. Este novo atributo será utilizado para a apresentação do mapa geográfico dos indicadores de caminhabilidade descrita na seção seguinte.

\subsubsection{Representação Geográfica dos Indicadores de Caminhabilidade}

Na próxima transição do iWalk, é apresentado um mapa geográfico representando a região e os segmentos que estão sendo analisadas pelo iWalk. A representação geográfica dos 
valores obtidos para cada segmento, de acordo com uma escala de 0 a 100 do indicador de caminhabilidade calculado é representada em uma escala de cores que vai do verde ao vermelho e simboliza a qualidade da infraestrutura analisada da seguinte maneira:

- Verde: de 81 a 100, caminhabilidade ótima

- Verde claro: de 61 a 80,99, caminhabilidade boa.

- Amarelo: de 41 a 60,99, caminhabilidade regular.

- Laranja: de 21 a 40,99, caminhabilidade ruim.

- Vermelho: de 0 a 20,99, caminhabilidade péssima.

No exemplo do estudo de caso realizado para a capital portuguesa, o iWalk apresenta a representação geográfica do indicador de caminhabilidade da topografia do bairro Belém, em Lisboa, conforme apresentado na Figura 3.

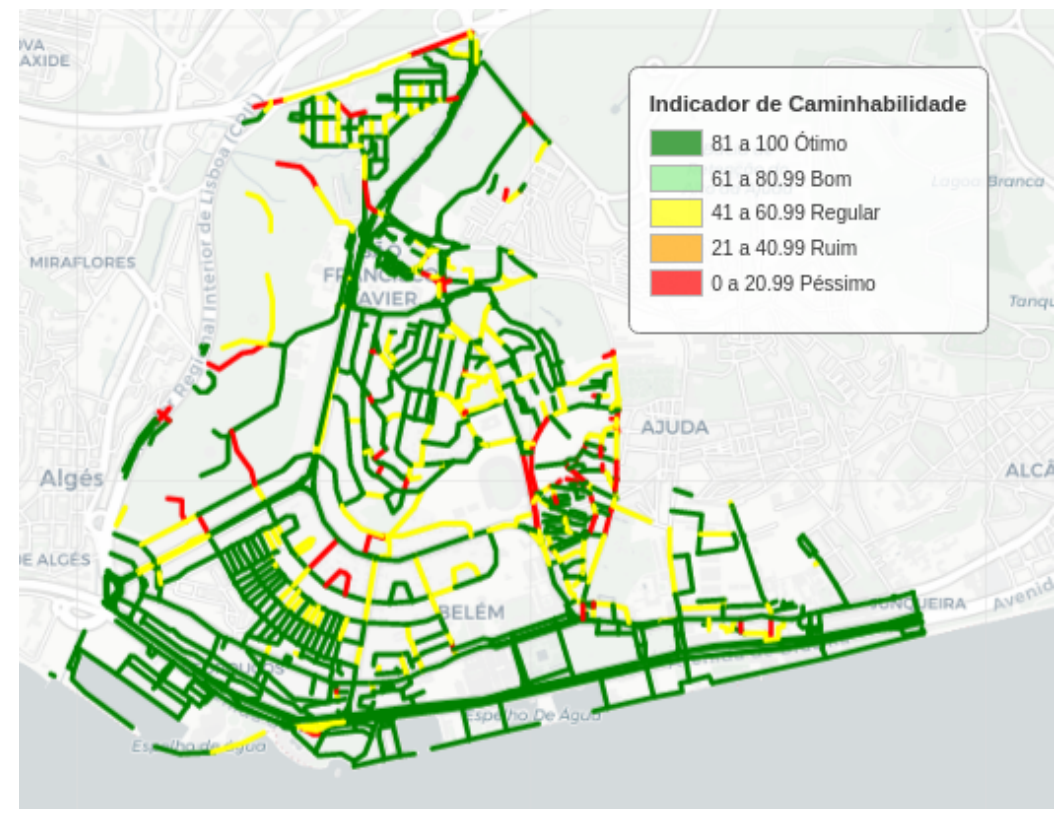

Figura 3. Mapa geográfico apresentado pelo iWalk para o indicador topográfico de declividade do bairro Belém, em Lisboa

\subsubsection{Cálculo do Indicador de Caminhabilidade da Área Total}

Na transição seguinte, com os valores obtidos por meio da normalização dos valores calculados das faixas de caminhabilidade, é obtida uma média geral dos valores (ME) calculados para cada segmento, para representar a valor do indicador de caminhabilidade da área total que está sendo analisada. Este cálculo é realizado utilizando a Equação 3, onde $N$ é o número de segmentos que foram calculados e $R i$ é o valor do indicador de caminhabilidade calculado pelo iWalk para cada segmento. Por exemplo, no estudo de caso realizado em Lisboa, o resultado obtido da média calculada para o bairro de Belém foi de 84,2 e a partir da mesma escala gráfica utilizada na representação geográfica, pode ser considerada ótima, pois se encontra dentro da faixa de 81 a 100.

$$
M E=\frac{\sum_{i=1}^{N} R i}{N}
$$




\section{Validação da Proposta}

Em um segundo momento, foram conduzidos experimentos com o objetivo de comparar o desempenho da abordagem proposta iWalk com um trabalho conhecido da literatura. A abordagem realizada em [Cardoso et al. 2019] é recente e utiliza uma grande variedade de técnicas para criar um índice de caminhabilidade aplicado à cidade de Belo Horizonte, por meio de um estudo de caso. Tendo em vista ainda que diferentes bases de dados abertos estão disponíveis no portal governamental da prefeitura de Belo Horizonte ${ }^{4}$, este estudo foi selecionado como base de comparação para a solução proposta iWalk.

\section{1. Índice e Indicadores de Caminhabilidade}

O índice de caminhabilidade proposto em [Cardoso et al. 2019] é um conjunto composto por diferentes indicadores que foram classificados em temas de maior abrangência. Na Tabela 1 é apresentada a seleção de indicadores que compõem o índice de caminhabilidade para Belo Horizonte. Um código de identificação (ID) foi estabelecido para cada atributo e serão utilizados também para identificação do indicador calculado pelo iWalk.

\begin{tabular}{|c|c|c|}
\hline CATEGORIAS & INDICADORES & ID \\
\hline \multirow{5}{*}{ Acessibilidade } & Largura efetiva do passeio & al \\
\hline & Conservação do Pavimento da calçada & $\mathrm{a} 2$ \\
\hline & Desníveis na calçada & a3 \\
\hline & Sinalização tátil & $\mathrm{a} 4$ \\
\hline & Topografia & a5 \\
\hline \multirow{5}{*}{ Atratividade } & Atratividade visual & bl \\
\hline & Permeabilidade visual & b2 \\
\hline & Arborização & b3 \\
\hline & Limpeza & b4 \\
\hline & Poluição & b5 \\
\hline \multirow{3}{*}{ Conectividade } & Tamanho da quadra & $\mathrm{c} 1$ \\
\hline & Acesso ao Transporte Público & $\mathrm{c} 2$ \\
\hline & Infraestrutura cicloviária & c3 \\
\hline \multirow{3}{*}{ Conforto } & Bancos para assentar & dl \\
\hline & Proteção contra intempéries & $\mathrm{d} 2$ \\
\hline & Infraestrutura Pluvial & $\mathrm{d} 3$ \\
\hline \multirow{2}{*}{ Segurança Pública } & Presença de pedestres (seguridade) & el \\
\hline & Iluminação & $\mathrm{e} 2$ \\
\hline \multirow{4}{*}{ Segurança Viária } & Possibilidade de conflito entre pedestres e veiculos na calçada & $\mathrm{f} 1$ \\
\hline & Barreira de proteção (buffers) & $\mathrm{f} 2$ \\
\hline & Largura do leito carroçável & $\mathrm{f3}$ \\
\hline & Velocidade dos veículos & $\mathrm{f} 4$ \\
\hline \multirow{2}{*}{ Uso do Solo } & Uso misto do solo & g1 \\
\hline & Parques e áreas verdes a uma distância caminhável & $\mathrm{g} 2$ \\
\hline \multirow{3}{*}{ Travessia } & Acesso à travessia & $\mathrm{hl}$ \\
\hline & Número de ruas na interseção & h2 \\
\hline & Sinalização na travessia & $\mathrm{h} 3$ \\
\hline
\end{tabular}

Tabela 1. Índice de Caminhabilidade de Belo Horizonte [Cardoso et al. 2019].

Em [Cardoso et al. 2019] foi atribuída uma nota com o nível de desempenho de cada indicador. As equações utilizadas neste trabalho de 2019 para o cálculo dos indicadores foram inseridos como parâmetros no iWalk, de modo a se realizar o mesmo cálculo de modo computacional e utilizando os dados abertos disponíveis, sem a necessidade das visitas presenciais à área de estudo.

\footnotetext{
${ }^{4}$ https://dados.pbh.gov.br/
} 


\section{2. Área de Estudo}

A mesma área selecionada por [Cardoso et al. 2019] foi utilizada para a validação do iWalk e trata-se de um trecho da rua Padre Eustáquio, na regional Noroeste da cidade de Belo Horizonte, incluindo seu entorno. Para inserir a área no iWalk, criou-se um novo arquivo GeoJSON com os dados geográficos dos limites da área indicada no estudo de referência. Na Figura 4, é possível observar a área do estudo de caso proposto em [Cardoso et al. 2019] (a) e (b), a mesma área representada pelo iWalk.

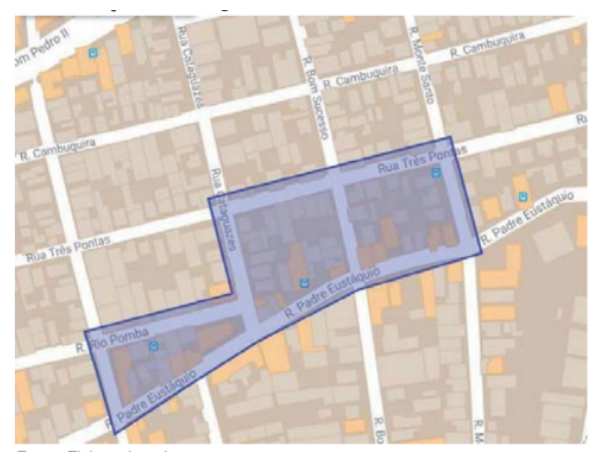

(a) Área de estudo em [Cardoso et al. 2019]

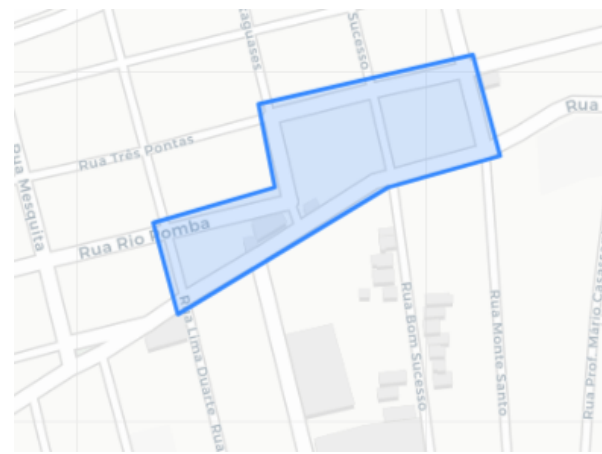

(b) Área de estudo representada no iWalk

Figura 4. Identificação da área de estudo

\subsection{Indicador de Caminhabilidade}

Para validação da proposta, analisou-se, no iWalk, um dos 27 indicadores analisados no trabalho de 2019. O indicador utilizado para a validação do iWalk foi a Topografia, presente na categoria Acessibilidade, identificado na Tabela 1 pelo identificador $a 5$ e que está presente na base de dados Declividade Trecho Logradouro, no portal de dados abertos da Prefeitura de Belo Horizonte. Utilizamos a base de dados que contém os limites geográficos da área de estudo descritos na seção anterior e executamos o fluxo completo do iWalk para calcular o indicador de caminhabilidade validado neste estudo. Na Figura 5, temos a representação geográfica dos resultados encontrados em [Cardoso et al. 2019] (a) e (b) a representação dos resultados encontrados no iWalk.

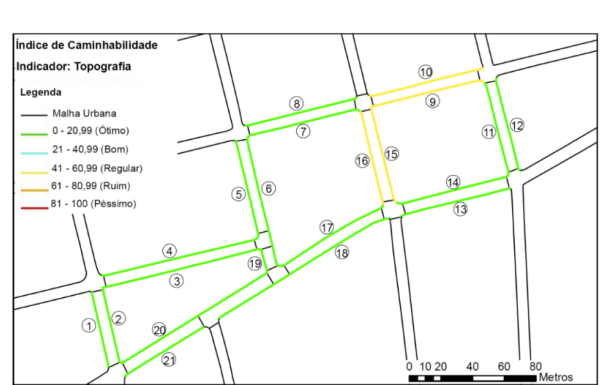

(a) Indicador Topografia em [Cardoso et al. 2019]

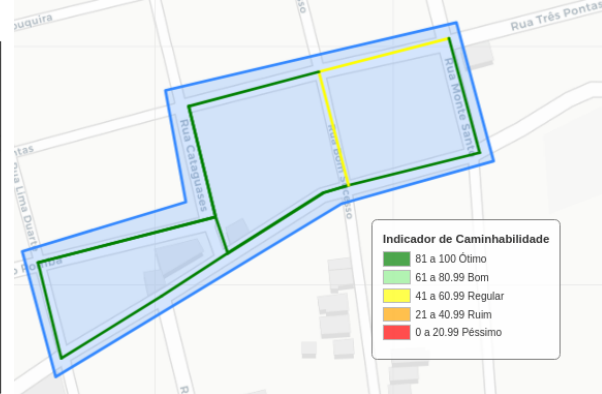

(b) Indicador de Topografia representado no iWalk

Figura 5. Representação geográfica dos resultados obtidos para o indicador de caminhabilidade Topografia 


\section{Conclusão e Trabalhos Futuros}

Neste trabalho, foi apresentada a solução iWalk para realizar o cálculo de um indicador de caminhabilidade de um determinado espaço público e permitir a consulta de indicadores que suportem decisões seja de cunho governamental ou pessoal utilizando dados abertos.

A solução se destaca por utilizar dados abertos disponíveis na internet, de maneira escalável e dispensando a necessidade de visitas in loco e busca ampliar o conhecimento dos fatores que podem influenciar na caminhabilidade. Para realizar o cálculo dos indicadores de caminhabilidade, o iWalk utiliza os atributos espaciais e não espaciais presentes nas bases de dados GeoJSON disponibilizados por portais governamentais de dados abertos. O iWalk realiza os cálculos por meio de um fluxo de operações que recebe como parâmetros iniciais a base de dados que contém os limites geográficos da área a ser estudada, a base que contém os dados dos indicadores a serem analisados, bem como as funções de para o cálculo do indicador de caminhabilidade de cada segmento analisado e a função para determinar a faixa de caminhabilidade em que o indicador se encontra.

Por meio de uma validação da proposta com um trabalho conhecido da literatura, o iWalk se apresentou como uma solução eficiente e escalável para realizar o cálculo de indicadores de caminhabilidade de espaços públicos. É eficiente pois é capaz de realizar o cálculo dos sem a necessidade de visitar a área de estudo presencialmente e é escalável pois a área de estudo a ser analisada é delimitada pelo polígono extraído na primeira iteração do iWalk, se este polígono for de uma área maior ou distinta da determinada anteriormente, o iWalk é capaz de calcular o indicador de caminhabilidade para toda a área selecionada, provendo conhecimento importante para tomada de decisão em projetos de redes compartilhadas e aplicações móveis.

Como trabalhos futuros, poderão ser calculados outros indicadores de caminhabilidade como Arborização, Iluminação, Largura do Leito Carroçável e Uso Misto do Solo, a fim de enriquecer os resultados. Desta forma, o iWalk poderá inserir novos indicadores de caminhabilidade, o que ampliará ainda mais sua efetividade.

\section{Agradecimentos}

Os autores agradecem o apoio à FAPEMIG (PPM-XII) e à Coordenação de Aperfeiçoamento de Pessoal de Nível Superior - Brasil (CAPES) - Código de Financiamento 001 .

\section{Referências}

Abnt, N. (2015). 9050. acessibilidade a edificações, mobiliário, espaços e equipamentos urbanos. Rio de Janeiro: Associação Brasileira de Normas Técnicas.

Albino, V., Berardi, U., and Dangelico, R. M. (2015). Smart cities: Definitions, dimensions, performance, and initiatives. Journal of urban technology, 22(1):3-21.

Bhadra, S., Sazid, A. T., and Esraz-Ul-Zannat, M. (2015). An objective assessment of walkability in khulna city: A gis based approach. In 2015 3rd International Conference on Green Energy and Technology (ICGET), pages 1-5. IEEE.

Cardoso, L., de Carvalho, I. R. V., and Nunes, N. T. R. (2019). Caminhabilidade como instrumento de mobilidade urbana: reflexões sobre a realidade de belo horizonte. $R e$ vista dos Transportes Públicos-ANTP-Ano, 41:2. 
Eastman, J. and Jiang, H. (1996). Fuzzy measures in multi-criteria evaluation. United States Department of Agriculture Forest Service General Technical Report RM, pages 527-534.

Fonseca, F., Ribeiro, P., Jabbari, M., Petrova, E., Papageorgiou, G., Conticelli, E., Tondelli, S., and Ramos, R. (2019). Smart pedestrian network: an integrated conceptual model for improving walkability. In International Conference on Society with Future: Smart and Liveable Cities, pages 125-142. Springer.

Forsyth, A. (2015). What is a walkable place? the walkability debate in urban design. Urban design international, 20(4):274-292.

Frank, L. D., Schmid, T. L., Sallis, J. F., Chapman, J., and Saelens, B. E. (2005). Linking objectively measured physical activity with objectively measured urban form: findings from smartraq. American journal of preventive medicine, 28(2):117-125.

Glazier, R., Weyman, J., Creatore, M., Gozdyra, P., Moineddin, R., Matheson, F., and Booth, G. (2012). Development and validation of an urban walkability index for toronto, canada. Toronto Community Health Profiles Partnership, pages 1-21.

Kuzmyak, J. R., Baber, C., and Savory, D. (2006). Use of walk opportunities index to quantify local accessibility. Transportation Research Record, 1977(1):145-153.

Leslie, E., Coffee, N., Frank, L., Owen, N., Bauman, A., and Hugo, G. (2007). Walkability of local communities: using geographic information systems to objectively assess relevant environmental attributes. Health \& place, 13(1):111-122.

Manaugh, K. and El-Geneidy, A. (2011). Validating walkability indices: How do different households respond to the walkability of their neighborhood? Transportation research part D: transport and environment, 16(4):309-315.

Mantri, A. (2008). A GIS based approach to measure walkability of a neighborhood. $\mathrm{PhD}$ thesis, University of Cincinnati.

Papageorgiou, G., Hadjigeorgiou, K., and Ness, A. N. (2020). An innovative way to promote walking via a smartphone pedestrian navigation application. In 2020 European Navigation Conference (ENC), pages 1-9. IEEE.

Quercia, D., Aiello, L. M., Schifanella, R., and Davies, A. (2015). The digital life of walkable streets. In Proceedings of the 24th international conference on World Wide Web, pages 875-884.

Schenk, T. (2015). Building a government data strategy: aligning open data and advanced research with the public and open science principles. In 2015 Second International Conference on eDemocracy \& eGovernment (ICEDEG), pages 18-18. IEEE.

Wang, D. and Song, C. (2015). Impact of human mobility on social networks. Journal of Communications and Networks, 17(2):100-109.

Xavier, W. Z. (2019). openmodal:uma plataforma para analise de servi cos de mobilidade urbana.

Zheng, Y., Capra, L., Wolfson, O., and Yang, H. (2014). Urban computing: concepts, methodologies, and applications. ACM Transactions on Intelligent Systems and Technology (TIST), 5(3):1-55. 